Article

\title{
Squeezing the Parameter Space for Lorentz Violation in the Neutrino Sector with Additional Decay Channels
}

\author{
Ulrich D. Jentschura $1,2,3$ (D) \\ 1 Department of Physics, Missouri University of Science and Technology, Rolla, MO 65409, USA; ulj@mst.edu \\ MTA-DE Particle Physics Research Group, P.O. Box 51, H-4001 Debrecen, Hungary \\ 3 MTA Atomki, P.O. Box 51, H-4001 Debrecen, Hungary
}

Received: 23 July 2020; Accepted: 24 August 2020; Published: 26 August 2020

check for updates

\begin{abstract}
The hypothesis of Lorentz violation in the neutrino sector has intrigued scientists for the last two to three decades. A number of theoretical arguments support the emergence of such violations, first and foremost for neutrinos, which constitute the "most elusive" and "least interacting" particles known to mankind. It is of obvious interest to place stringent bounds on the Lorentz-violating parameters in the neutrino sector. In the past, the most stringent bounds have been placed by calculating the probability of neutrino decay into a lepton pair, a process made kinematically feasible by Lorentz violation in the neutrino sector, above a certain threshold. However, even more stringent bounds can be placed on the Lorentz-violating parameters if one takes into account, additionally, the possibility of neutrino splitting, i.e., of neutrino decay into a neutrino of lower energy, accompanied by "neutrino-pair Čerenkov radiation." This process has a negligible threshold and can be used to improve the bounds on Lorentz-violating parameters in the neutrino sector. Finally, we take the opportunity to discuss the relation of Lorentz and gauge symmetry breaking, with a special emphasis on the theoretical models employed in our calculations.
\end{abstract}

Keywords: lorentz violation; neutrinos; gauge invariance; mass mixing; icecube detector; physics beyond the standard models

\section{Introduction}

Neutrinos are the most elusive particles within the standard model of elementary interactions. Speculation about their tachyonic nature started with reference [1], and has led to the development of a few interesting scenarios [2]. Within the Lorentz-violating scenarios [3-13], many different tensor structures involving Lorentz-violating parameters can be pursued. Here, we assume that, in a preferred particular (observer) Lorentz frame, an isotropic dispersion relation of the form $E=\sqrt{\vec{p}^{2} v^{2}+m^{2}}$ with $v>1$ holds. (In this article, we use physical units with $\hbar=\epsilon_{0}=c=1$ ). Formalizing the Lorentz-violating ideas, the Lorentz-violating extension of the standard model (SME) was developed with strong inspiration from string theory [14,15].

Kinematically, decay among neutrino mass eigenstates is allowed due to their mass differences, while decay rates for "ordinary" neutrinos within the standard model formalism (for both Dirac as well as Majorana) exceed the lifetime of Universe by orders of magnitude. Lorentz-violating neutrinos undergo stronger decay and energy loss mechanisms than "ordinary" neutrinos because of their dispersion relation $E=\sqrt{\vec{p}^{2} v^{2}+m^{2}} \approx|\vec{p}| v$ (at high energy), which makes a number of decay channels (without GIM suppression; see references $[16,17])$ kinematically possible.

Some remarks on the origin of modified dispersion relations of the form $E=\sqrt{\vec{p}^{2} v^{2}+m^{2}} \approx|\vec{p}| v$ might be in order. Modified dispersion relations could in principle be induced by modified theories 
of gravity, and modifications of the Einstein equivalence principle. In reference [18], it has been suggested that the invariant arena for nonquantum physics is a phase space rather than spacetime, and the locality of an even in space-time is replaced by relative locality in which different observers see different spacetimes, and the spacetimes they observe are energy and momentum dependent. This hypothesis can lead to a modified dispersion relation of the kind investigated here. Effects due to quantum gravity may also induce modified dispersion relations $[19,20]$. In a different context, Lorentz breaking induced at the Planck scale may also induce such relations [21-23], in the sense of "doubly special relativity," which works on the assumption that dynamics are governed by two observer-independent quantities, the speed of light and an additional constant energy scale, which could be the Planck energy scale (see also reference [24]). The phenomenological consequences of tiny Lorentz violations, which are rotationally and translationally invariant in a preferred frame, and are renormalizable while preserving anomaly cancellation and gauge invariance under the standard model gauge group $S U(3) \otimes S U(2) \otimes U(1)$, have been analyzed in reference [25].

There are a number of phenomena which could direct us to have a look at the neutrino sector for Lorentz violation. Namely, for example, the early arrival of neutrinos from the 1987 supernova still inspires (some) physicists. Specifically, under the Mont Blanc, in the early morning hours of 23 February 1987, a shower of neutrinos of interstellar origin arrived about six hours earlier then the visible light from the Siderius Nuntius SN1987A supernova. This event has been recorded in reference [26], and it was asserted that such an event could happen by accident once in about 1000 years. Direct measurements of neutrino velocities have given results that are consistent with the speed of light within experimental uncertainty, but with the experimental result being a littler larger than the speed of light. For example, the MINOS experiment [27] has measured superluminal neutrino propagation velocities which differ from the speed of light by a relative factor of $(5.1 \pm 2.9) \times 10^{-5}$ at an energy of about $E_{v} \approx 3 \mathrm{GeV}$, compatible with an earlier FERMILAB experiment [28]. Furthermore, neutrinos cannot be used to transmit information (at least not easily) because of their small interaction cross sections. Superluminality of neutrinos would thus not necessarily lead to violation of causality at a macroscopic level, as demonstrated in Appendix A.2 of reference [29]. Similar arguments have been made in reference [30], where it was shown that problems with microcausality, in Lorentz-violating theories, are alleviated for small Lorentz-violating parameters and in so-called concordant frames where the boost velocities are not too large. For neutrinos, corresponding problems are further alleviated by the fact that their interaction cross sections are small; hence, it becomes very hard to transport information superluminally even if the dispersion relation indicates such effects (see also Appendix A.2 of reference [29]).

We should also note that, when Lorentz invariance is violated, superluminality does not necessarily lead to problems. Under certain additional assumptions, causality arguments related to superluminal signal propagation simply do not apply in this case. For example, if photons themselves propagate faster than $c$ (the limiting velocity of massive standard fermions) in some Lorentz-violating theories, then, as long as information propagates along or inside the modified lightcone (as defined by a modified effective metric), it can propagate faster than $c$ without implying causality issues (see, e.g., the paragraph around Equation (9) in reference [31]). Further information on this point can be found in references [32-36]. E.g., reference [32] provides information on (micro)causality problems for the purely timelike case of Maxwell-Chern-Simons theory (operator of dimension 3 for photons).

Some more explanatory remarks on gauge invariance and Lorentz violation are probably in order. One might argue that gauge invariance should be seen as the guiding principle to make consistent a nonabelian gauge interaction mediated by spin-one massive particles through the (experimentally confirmed) Higgs mechanism, and that gauge invariance should be retained at all cost, even if Lorentz symmetry is broken. However, this demand overlooks two aspects. The first is that the origin of the Lorentz-violating terms could be assumed to be commensurate with the Planck scale [14,21,24], in which case it is questionable if our usual concepts of gauge invariance could be transported without changes to the extreme energy scales; in any case, we would assume the standard model gauge group, 
at the energy scale relevant to Lorentz violation, to be replaced by a unified gauge group, e.g., $\mathrm{SO}(1,13)$ (see references [37-41]). Further fundamental modifications of the gauge principle at extreme energy scales are also conceivable. The second and more important overlooked aspect is that Lorentz violation constitutes a form of gauge invariance violation. Namely, Einstein's theory of general relativity is the classical, gauged theory of gravitation, in which the global Lorentz symmetry is elevated to a gauged, local symmetry [42]. This observation offers the construction principle for the spin connection of Dirac fields in curved space-times, which has been explored in recent monographs [42] and papers [43,44]. To reemphasize the point, we recall that Lorentz invariance violation is gauge invariance violation within General Relativity [42].

We organized the paper as follows. After consideration of the calculation of the threshold for the superluminal decay processes (Section 2), we outline the calculations of the decay and energy loss rates in Section 3, before discussing an attractive scenario for Lorentz violation in the neutrino sector, and its signatures, in Section 4. Conclusions are reserved for Section 5.

\section{Threshold Considerations}

We refer to the lepton-pair Čerenkov radiation process (LPCR) in Figure 1a, and the neutrino-pair Čerenkov radiation process (NPCR) is depicted in Figure $1 \mathrm{~b}$. In order to make neutrino decay kinematically possible, it is necessary to fulfill certain threshold conditions. Let us denote the outgoing fermions in the generic decay processes depicted in Figure 1 by

$$
v \rightarrow v+f+\bar{f},
$$

with a pair of a massive fermion $f$ and its antiparticle $\bar{f}$ being emitted in the process.

Energy-momentum conservation implies that (in the notation of Figure 1)

$$
\left(p_{1}-p_{3}\right)^{2}=q^{2}=\left(p_{2}+p_{4}\right)^{2} .
$$

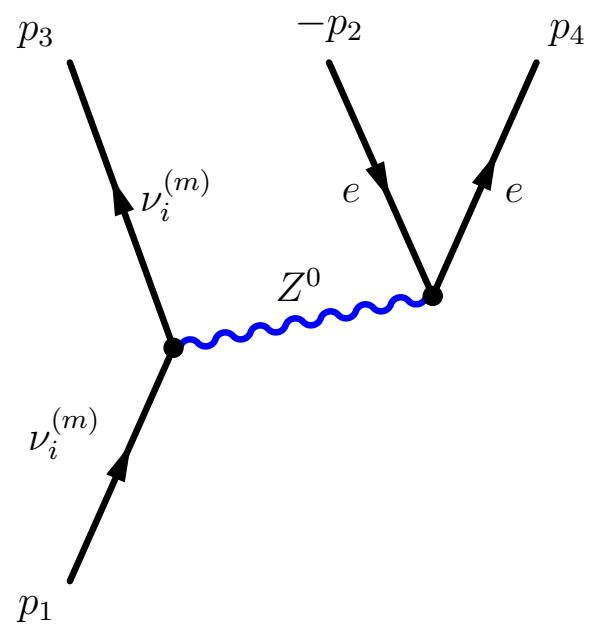

(a)

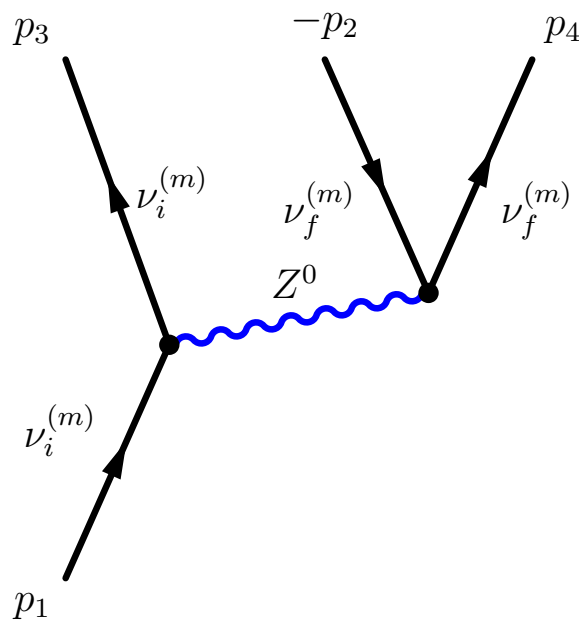

(b)

Figure 1. In the lepton-pair Čerenkov radiation process (a), an oncoming Lorentz-violating initial neutrino mass eigenstate $v_{i}^{(m)}$ decays, under emission of a virtual $Z^{0}$ boson, into an electron-positron pair. The sum of the outgoing pair momenta is $p_{2}+p_{4}$; one observes an inverted direction of the fermionic antiparticle line. The arrow of time is from bottom to top. The (blue) bosonic line carries the four-momentum $q$. Diagram (b) describes the neutrino-pair Čerenkov radiation process, with a final neutrino mass eigenstate $v_{f}^{(m)}$. 
Let us first consider the case of a massive outgoing pair $2+4$, with rest mass $m_{f}$, and vanishing Lorentz-violating parameter. Threshold is reached for collinear emission geometry. The incoming four-momentum is $p_{1}=\left(E_{1}, \vec{p}_{1}\right)$, where $E_{1}=\sqrt{\vec{p}_{1}^{2} v_{i}^{2}+m_{v}^{2}} \approx\left|\vec{p}_{1}\right| v_{i}$, and $m_{v}$ is the neutrino mass. Assuming an incoming neutrino energy well above its rest mass, we can do this approximation. By contrast, one has $p_{3}=(0, \overrightarrow{0})$, so that the total four-momentum transfer $q$ goes into the pair.

A few remarks on the dispersion relations used in the current paper, are in order. We can observe that the relation $E_{1}=\sqrt{\vec{p}_{1}^{2} v_{i}^{2}+m_{v}^{2}}$ is isotropic, and it induces superluminal velocities. However, it should be noted that the modified dispersion relation applied to neutrino sector can introduce a rich phenomenology even in other sectors, as oscillations, without requiring superluminal velocities for $v_{i}>1$. Many models can be explored (see references $[5-7,9,45-48]$ ). As explained above, here we assume a simple form for the modified dispersion relation, applicable at least in a preferred observer Lorentz frame.

Returning to the discussion of the threshold, we observe that, for collinear geometry, one has $p_{2}^{\mu}=p_{4}^{\mu}=\left(E_{f}, \vec{p}_{f}\right)$, where $E_{f}=\sqrt{\vec{p}_{f}^{2}+m^{2}}$. Under these assumptions, $p_{2}+p_{4}=\left(2 \sqrt{\vec{p}_{f}^{2}+m^{2}}, 2 \vec{p}_{f}\right)$, so that $\left(p_{2}+p_{4}\right)^{2}=4 m_{f}^{2}$. The threshold condition becomes

$$
p_{1}^{2}\left(v_{i}^{2}-1\right) \geq 4 m_{f}^{2}, \quad p_{1} \approx E_{1} \geq \frac{2 m_{f}}{\sqrt{v_{i}^{2}-1}}=\frac{2 m_{f}}{\sqrt{\delta_{i}}} .
$$

Here,

$$
v_{i}=\sqrt{1+\delta_{i}} .
$$

The threshold condition $E_{\text {th }}=2 m_{f} / \sqrt{\delta_{i}}$ has been used extensively in references [49-51]. Equation (3) implies that the threshold for NPCR is lower by at a least six orders of magnitude as compared to LPCR.

The kinematic considerations are very different in the high-energy regime, when both the incoming (decaying) particles and the outgoing particles are Lorentz violating. Masses can be neglected. In this case, one has at threshold $p_{2}=p_{4}=\left(E_{f}, \vec{p}_{f}\right)$, where $E_{f}=\left|\vec{p}_{f}\right| v_{f}$, so that at threshold

$$
p_{1}^{2}\left(v_{i}^{2}-1\right) \geq 4 p_{f}^{2}\left(v_{f}^{2}-1\right),
$$

Due to equipartition of the energy among each particle of the outgoing pair at threshold, one has $p_{f} \approx E_{f}=E_{1} / 2 \approx p_{1} / 2$. In this case, the threshold condition reduces to

$$
\left(v_{i}^{2}-1\right) \geq\left(v_{f}^{2}-1\right), \quad \delta_{i}>\delta_{f} .
$$

Here, $v_{f}=\sqrt{1+\delta_{f}}$. For $\delta_{i}=\delta_{f}$, no phase space is available in order to accommodate for the decay. This consideration explains why all results communicated in reference [51] display a factor $\delta_{i}-\delta_{f}$; decay takes place from "faster" to "slower" mass eigenstates.

\section{Outline of the Calculation}

The understanding of decay processes involving Lorentz-violation has been advanced through references [49-51]. Let us briefly recall elements of the derivation given in reference [51]. One particular question is how to express the decay rate for an (initially) flavor-eigenstate neutrino (the electroweak Lagrangian is flavor-diagonal) in terms of mass eigenstates. We have, in the same obvious notation as used in reference [51],

$$
v_{k}^{(f)}=\sum_{\ell} U_{k \ell} v_{\ell}^{(m)}
$$


with the Pontecorvo-Maki-Nakagawa-Sakata (PMNS) matrix $U_{k \ell}$. The interaction interaction $\mathcal{L}_{W}$ with the $Z^{0}$ boson in the flavor basis is

$$
\mathcal{L}_{W}=-\frac{g_{w}}{4 \cos \theta_{W}} \sum_{k} \bar{v}_{k}^{(f)} \gamma^{\mu}\left(1-\gamma^{5}\right) v_{k}^{(f)} Z_{\mu} .
$$

Here, $g_{w}$ is the weak coupling constant, and $\theta_{W}$ is the Weinberg angle. A unitary transformation leads to

$$
\mathcal{L}=-\frac{g_{w}}{4 \cos \theta_{W}} \sum_{k, \ell, \ell^{\prime}} U_{\ell k}^{+} U_{k \ell^{\prime}} \bar{v}_{\ell}^{(m)} \gamma^{\mu}\left(1-\gamma^{5}\right) v_{\ell^{\prime}}^{(m)} Z_{\mu} .
$$

The interaction with the $Z^{0}$ boson in the mass eigenstate basis therefore reads as follows:

$$
\mathcal{L}=-\frac{g_{w}}{4 \cos \theta_{W}} \sum_{\ell} \bar{v}_{\ell}^{(m)} \gamma^{\mu}\left(1-\gamma^{5}\right) v_{\ell}^{(m)} Z_{\mu}
$$

In order to model the free Lorentz-violating neutrino Lagrangian, one introduces an effective metric with a tilde:

$$
\mathcal{L}=\sum_{\ell} \mathrm{i} \bar{v}_{\ell}^{(m)} \gamma^{\mu}\left(1-\gamma^{5}\right) \tilde{g}_{\mu v}\left(v_{\ell}\right) \partial^{v} v_{\ell}^{(m)}
$$

The modified metric defines a modified light cone according to $\tilde{g}_{\mu v}\left(v_{\ell}\right) k^{\mu} k^{v}=0$. Here,

$$
\tilde{g}_{\mu v}\left(v_{\ell}\right)=\operatorname{diag}\left(1,-v_{\ell},-v_{\ell},-v_{\ell}\right) \text {. }
$$

The dispersion relation

$$
E_{\ell}=|\vec{p}| v_{\ell}
$$

follows as the massless limit of $E_{\ell}=\sqrt{\left(|\vec{p}| v_{\ell}\right)^{2}+m_{\ell}^{2}}$. For neutrinos, we know that the $m_{\ell}$ terms are different. Thus, there is reason to assume that the $\delta_{\ell}=\sqrt{v_{\ell}^{2}-1}$ terms are also different among mass (flavor) eigenstates, if they are nonvanishing.

One defines parameter $v_{\text {int }}$ and $\delta_{\text {int }}$ by the relation

$$
v_{\text {int }}=\sqrt{1+\delta_{\text {int }}}
$$

for the unified description of LPCR and NPCR; the effective four-fermion Lagrangian for the process reads as

$$
\mathcal{L}_{\text {int }}=f_{e} \frac{G_{F}}{2 \sqrt{2}} \bar{v}_{i}^{(m)} \gamma^{\lambda}\left(1-\gamma^{5}\right) v_{i}^{(m)} \tilde{g}_{\lambda \sigma}\left(v_{\text {int }}\right) \bar{\psi}_{f} \gamma^{\sigma}\left(c_{V}-c_{A} \gamma^{5}\right) \psi_{f} .
$$

Cohen and Glashow [49] set $v_{\text {int }}=1$. (In reference [51], on a number of occasions, the parameter used in reference [49] had been inadvertently indicated as $v_{\text {int }}=0$, which is not the case. We take the opportunity to point out that of course, the parameter $v_{\text {int }}=1$ implies that $\delta_{\text {int }}=0$, which was the intended statement in reference [49].) Bezrukov and Lee [50] used the parameters $v_{\text {int }}=1$ ("model I") and $v_{\text {int }}=v_{i}$ ("model II"). In reference [51], the parameter $v_{\text {int }}$ is kept as a variable. As explained in detail in reference [52], "gauge invariance" (with respect to a restricted subgroup of the electroweak sector) can be restored if one uses the value $v_{\text {int }}=v_{i} v_{f}$. Both Cohen and Glashow [49], and Bezrukov and Lee [50], assumed that $\delta_{f}=0$ for LPCR. The parameter $f_{e}$ characterizes the process:

$$
f_{e}=\left\{\begin{array}{ll}
1, & \psi_{f}=v_{f}^{(m)} \\
2, & \psi_{f}=e
\end{array} .\right.
$$


Approximately, one has

$$
\left(c_{V}, c_{A}\right)=\left\{\begin{array}{ll}
(1,1) & \psi_{f}=v_{f}^{(m)} \\
\left(0,-\frac{1}{2}\right), & \psi_{f}=e
\end{array} .\right.
$$

The characteristic matrix element is

$$
\mathcal{M}=f_{e} \frac{G_{F}}{2 \sqrt{2}}\left[\bar{u}_{i}\left(p_{3}\right) \gamma^{\lambda}\left(1-\gamma^{5}\right) u_{i}\left(p_{1}\right)\right] \tilde{g}_{\lambda \sigma}\left(v_{\text {int }}\right)\left[\bar{u}_{f}\left(p_{4}\right)\left(c_{V} \gamma^{\sigma}-c_{A} \gamma^{\sigma} \gamma^{5}\right) v_{f}\left(p_{2}\right)\right] .
$$

Key to the calculation is the fact that one can split the three-particle outgoing phase space

$$
\begin{aligned}
\Gamma & =\frac{1}{2 E_{1}} \int \mathrm{d} \phi_{3}\left(p_{2}, p_{3}, p_{4} ; p_{1}\right) \frac{1}{n_{s}} \sum_{\text {spins }}|\mathcal{M}|^{2} \\
& =\frac{1}{2 E_{1}} \int_{M_{\min }^{2}}^{M_{\max }^{2}} \frac{\mathrm{d} M^{2}}{2 \pi} \mathrm{d} \phi_{2}\left(p_{3}, p_{24} ; p_{1}\right) \mathrm{d} \phi_{2}\left(p_{2}, p_{4} ; p_{24}\right) \frac{1}{n_{s}} \sum_{\text {spins }}|\mathcal{M}|^{2},
\end{aligned}
$$

with appropriate limits for $M_{\min }^{2}$ and $M_{\max }^{2}$ being given as follows,

$$
M_{\min }^{2}=\delta_{f}\left(\left|\vec{p}_{2}\right|+\left|\vec{p}_{4}\right|\right)^{2}, \quad M_{\max }^{2}=\delta_{i}\left(\left|\vec{p}_{1}\right|-\left|\vec{p}_{3}\right|\right)^{2} .
$$

The following splitting relation for the phase space is crucial to a simplification of the integrations (for details, see reference [53] and Equation (43) of reference [51]),

$$
\begin{aligned}
& \mathrm{d} \phi_{3}\left(p_{2}, p_{3}, p_{4} ; p_{1}\right)=\int \frac{\mathrm{d} M^{2}}{2 \pi} \underbrace{\frac{\mathrm{d}^{4} p_{3}}{(2 \pi)^{3}} \delta_{+}\left(p_{3}^{2}-\delta_{i} k_{3}^{2}\right) \frac{\mathrm{d}^{4} p_{24}}{(2 \pi)^{3}} \delta_{+}\left(p_{24}^{2}-M^{2}\right)(2 \pi)^{4} \delta^{(4)}\left(p_{1}-p_{3}-p_{24}\right)}_{=\mathrm{d} \phi_{2}\left(p_{3}, p_{24} ; p_{1}\right)} \\
& \times \underbrace{\frac{\mathrm{d}^{4} p_{2}}{(2 \pi)^{3}} \delta_{+}\left(p_{2}^{2}-\delta_{f} k_{2}^{2}\right) \frac{\mathrm{d}^{4} p_{4}}{(2 \pi)^{3}} \delta_{+}\left(p_{4}^{2}-\delta_{f} k_{4}^{2}\right)(2 \pi)^{4} \delta^{(4)}\left(p_{24}-p_{2}-p_{4}\right)}_{=\mathrm{d} \phi_{2}\left(p_{2}, p_{4} ; p_{24}\right)} \\
& =\int \frac{\mathrm{d} M^{2}}{2 \pi} \mathrm{d} \phi_{2}\left(p_{3}, p_{24} ; p_{1}\right) \mathrm{d} \phi_{2}\left(p_{2}, p_{4} ; p_{24}\right) .
\end{aligned}
$$

Here, $p_{24}=p_{2}+p_{4}$, and we denote the spatial part of the four-vector $p_{i}^{\mu}$ as $\vec{k}_{i}$ with $i=1,2,3,4$, so that $p_{i}^{\mu}=\left(E_{i}, \vec{k}_{i}\right)$, and since $E_{i}=\left(1+\delta_{i}\right)\left|\vec{k}_{i}\right|$, one has $g^{\mu v} \tilde{g}_{\mu \alpha} \tilde{g}_{\nu \beta} p_{i}^{\alpha} p_{i}^{\beta}=g_{\mu \nu} p_{i}^{\mu} p_{i}^{v}-\delta_{i} k_{i}^{2}=p_{i}^{2}-\delta_{i} k_{i}^{2}$ (see also Equation (40) of reference [51]). This relation explains the argument of some of the Dirac- $\delta$ functions in Equation (21). The general result for the decay rate, unifying both processes depicted in Figure 1, reads as follows.

$$
\begin{aligned}
\Gamma_{v_{i} \rightarrow v_{i} \psi_{f} \bar{\psi}_{f}} & =\frac{G_{F}^{2} k_{1}^{5}}{192 \pi^{3}} f_{e}^{2} \frac{c_{V}^{2}+c_{A}^{2}}{420 n_{s}}\left(\delta_{i}-\delta_{f}\right)\left[\left(60-43 \sigma_{i}\right)\left(\delta_{i}-\delta_{f}\right)^{2}\right. \\
& +2\left(50-32 \sigma_{i}-25 \sigma_{f}+7 \sigma_{i} \sigma_{f}\right)\left(\delta_{i}-\delta_{f}\right) \delta_{f} \\
& \left.+7\left(4-3 \sigma_{i}-3 \sigma_{f}+2 \sigma_{i} \sigma_{f}\right) \delta_{f}^{2}+7 \delta_{\mathrm{int}}^{2}\right] .
\end{aligned}
$$

This result vanishes for $\delta_{i}=\delta_{f}$ (see the discussion in Section 2). Cohen and Glashow [49] have $n_{s}=2$ active spin states for the (initial) neutrino, while Bezrukov and Lee [50] calculate with $n_{s}=1$, implying that the authors of reference [50] assume that only one spin state exists in nature. This assumption affects the averaging over the initial quantum states involved in the process. The $\sigma$ parameters depend on the way in which spin polarization sums are carried out, 


$$
\sigma_{i}=\left\{\begin{array}{ll}
0, & \text { CG spin sum for } v_{i} \\
1, & \text { BL spin sum for } v_{i}
\end{array}, \sigma_{f}=\left\{\begin{array}{ll}
0, & \text { CG spin sum for } \psi_{f} \\
1, & \text { BL spin sum for } \psi_{f}
\end{array} .\right.\right.
$$

In reference [49], the Cohen-Glashow (CG) spin sum ("polarization sum") is simply taken as the standard spin sum for massless fermions.

$$
\sum_{s} v_{\ell, s} \otimes \bar{v}_{\ell, s}=p^{\mu} g_{\mu v} \gamma^{v}
$$

In reference [50], the Bezrukov-Lee (BL) spin sum is based on a somewhat more advanced treatment of the eigenspinors of superluminal neutrino mass eigenstates and reads as

$$
\sum_{s} v_{\ell, s} \otimes \bar{v}_{\ell, s}=p^{\mu} \tilde{g}_{\mu v}\left(v_{\ell}\right) \gamma^{v}
$$

The general result for the energy loss rate, applicable to both processes in Figure 1, reads as

$$
\begin{aligned}
& \frac{\mathrm{d} E_{v_{i} \rightarrow v_{i} \psi_{f} \bar{\psi}_{f}}}{\mathrm{~d} x}=-\frac{G_{F}^{2} k_{1}^{6}}{192 \pi^{3}} f_{e}^{2} \frac{c_{V}^{2}+c_{A}^{2}}{672 n_{s}}\left(\delta_{i}-\delta_{f}\right) \\
& \times\left[\left(75-53 \sigma_{i}\right)\left(\delta_{i}-\delta_{f}\right)^{2}+\left(122-77 \sigma_{i}-61 \sigma_{f}+16 \sigma_{i} \sigma_{f}\right)\left(\delta_{i}-\delta_{f}\right) \delta_{f}\right. \\
& \left.\quad+8\left(4-3 \sigma_{i}-3 \sigma_{f}+2 \sigma_{i} \sigma_{f}\right) \delta_{f}^{2}+8 \delta_{\mathrm{int}}^{2}\right] .
\end{aligned}
$$

In reference [51], we verified and checked compatibility with all formulas contained in references [49] and [50]. This is important because it confirms that the model dependence of the results is only contained in the numerical prefactors, but not in the overall scaling of the results.

As outlined in reference [51], one can parameterize the results for NPCR as follows.

$$
\Gamma_{v_{i} \rightarrow v_{i} v_{f} \bar{v}_{f}}=b \frac{G_{F}^{2}}{192 \pi^{3}} k_{1}^{5}, \quad \frac{\mathrm{d} E_{v_{i} \rightarrow v_{i} v_{f} \bar{v}_{f}}}{\mathrm{~d} x}=-b^{\prime} \frac{G_{F}^{2}}{192 \pi^{3}} k_{1}^{6} .
$$

For the CG spin sum, one obtains the following $b$ parameters,

$$
\begin{aligned}
& b_{\mathrm{CG}}=\frac{1}{7}\left(\delta_{i}-\delta_{f}\right)\left[\left(\delta_{i}-\delta_{f}\right)^{2}+\frac{5}{3} \delta_{f}\left(\delta_{i}-\delta_{f}\right)+\frac{7}{15} \delta_{f}^{2}\right], \\
& b_{\mathrm{CG}}^{\prime}=\frac{25}{224}\left(\delta_{i}-\delta_{f}\right)\left[\left(\delta_{i}-\delta_{f}\right)^{2}+\frac{112}{75} \delta_{f}\left(\delta_{i}-\delta_{f}\right)+\frac{32}{75} \delta_{f}^{2}\right] .
\end{aligned}
$$

For the BL spin sum, one obtains

$$
\begin{aligned}
& b_{\mathrm{BL}}=\frac{17}{210}\left(\delta_{i}-\delta_{f}\right)\left[\left(\delta_{i}-\delta_{f}\right)^{2}+\frac{7}{17} \delta_{\mathrm{int}}^{2}\right], \\
& b_{\mathrm{BL}}^{\prime}=\frac{11}{168}\left(\delta_{i}-\delta_{f}\right)\left[\left(\delta_{i}-\delta_{f}\right)^{2}+\frac{4}{11} \delta_{\mathrm{int}}^{2}\right] .
\end{aligned}
$$

Typically, one finds [51] numerical prefactors in these formulas are larger than those for LPCR by a factor of four or five. Also, NPCR has negligible threshold.

In papers of Stecker and Scully [12,54,55], the following bound was derived for the Lorentz-violating parameter of the electron-positron field alone (watch out for a difference in the conventions used for defining the $\delta_{e}$ parameter):

$$
\delta_{e} \leq 1.04 \times 10^{-20} .
$$


We should stress that this bound concerns oncoming electrons (not neutrinos!) and has nothing to do with the processes studied here.

The observation of very-high-energy neutrinos by IceCube, taking into consideration the LPCR process (but not NPCR!), implies that the Lorentz-violating parameter for neutrinos cannot be larger than (reference [12]):

$$
\delta_{v} \leq 2.0 \times 10^{-20} .
$$

This bound is based on the assumption that $\delta_{e}$ and $\delta_{v}$ are different. Colloquially speaking, we can say that, if $\delta_{v}$ were larger, then "Big Bird" (the $2 \mathrm{PeV}$ specimen found in IceCube, see references [56,57]) would have already decayed before it arrived at the IceCube detector. However, the full analysis requires Monte Carlo simulations involving astrophysical data and is much more involved [12,54,55].

Provided the Lorentz-violating parameters for the different neutrino mass eigenstates are different, low-energy neutrinos are affected by the decay and energy loss processes connected with NPCR, in view of a negligible threshold for NPCR. As already emphasized, typical numerical coefficients for NPCR are a factor of four or five larger than for LPCR, depending on the model used for the spin sums. This enhances the importance of the NPCR effect. Inspired by Equation (31), we thus conjecture here that a full analysis of astrophysical data, using the NPCR process as a limiting factor for the observation of high-energy neutrinos, should yield a bound in the order of

$$
\left|\delta_{i}-\delta_{f}\right| \leq \frac{1}{5^{1 / 3}} \times 2.0 \times 10^{-20} \sim 1.2 \times 10^{-21},
$$

where the prefactor takes into account the scaling of the effect with the $\delta$ parameter. Specifically, the decay and energy loss rates typically scale with the factor $\left(\delta_{i}-\delta_{f}\right)^{3}$. It would be very fruitful if this conjecture were to be checked against astrophysical data in an independent investigation.

\section{An Attractive Scenario}

At first, one might see a dilemma: Within a fully $S U(2)_{L}$ gauge-invariant theory, with uniform Lorentz-violating parameters over all particle generations, one necessarily has $\delta_{v}=\delta_{e}$ (see reference [52] for a detailed discussion), and so, the bound $\delta_{v} \leq 2.0 \times 10^{-20}$ given in Equation (31) is not applicable, because the LPCR process does not exist; but then, one has to acknowledge that the bound $\delta_{e} \leq 1.04 \times 10^{-20}$ given in Equation (30), which was originally derived for electrons, based on other physical processes, automatically also applies to the neutrino sector.

Thus, the dilemma is that either one has to give up gauge invariance and use different Lorentz-violating parameters for neutrinos, as opposed to charged leptons within the same particle generation, or, if one insists on gauge invariance, or, assume different Lorentz-violating parameters for different generations. Otherwise, the insistence on gauge invariance would defeat part of the purpose of looking at the neutrino sector for Lorentz violation. This is because in the latter case, for uniform Lorentz-violating parameters among all three generations, by assumption, the Lorentz-violating parameters for neutrinos and charged left-handed leptons within the same $S U(2)_{L}$ doublet are necessarily the same; the tight bounds on Lorentz-violating parameters in the charged-fermion sector automatically apply to the neutrino sector as well. (Here we ignore the somewhat remote possibility of different Lorentz violating parameters for the right-handed and left-handed sectors of one and the same generation). This observation has important consequences when examining the first-generation $S U(2)_{L}$ doublet, consisting of $\left(v_{e}, e_{L}\right)$. Electrons and positrons are stable particles, and small violations of Lorentz invariance would lead to violations of causality on a macroscopic level (see Appendix A.2 of reference [29]). (Note, also, that this statement does not hold if the limiting velocity for fermions turns out to be smaller as opposed to larger than the speed of light. Furthermore, there are conceivable modifications of Maxwell theory, as already discussed in Section 1, where causality violations are avoided due to modified light cones [31-36].) Conversely, if we had to carry over all restrictions on 
Lorentz-violating electron parameters to the electron neutrino sector, then this would nullify all the motivations listed in Section 1 for investigating the first-generation neutrino sector.

On the contrary, If one accepts the necessity that different Lorentz-violating parameters should be used for each of the three known particle generations, then one needs to acknowledge that the parameter space for differential Lorentz-violation among neutrino mass eigenstates is restricted by additional constraints due to the NPCR process [51]. An attractive gauge-invariant scenario could still be found, as follows. Namely, one might observe that, as per the discussion in Appendix A.2 of reference [29], causality violations due to Lorentz violation are less severe for unstable particles, which decay and therefore are not amenable to the reliable transport of information. Part of the above sketched dilemma could thus be avoided as follows. One first observes that, as per the above argument, problems with respect to causality are less severe in the second-generation $S U(2)_{L}$ doublet $\left(v_{\mu}, \mu_{L}\right)$ and also in the third-generation $S U(2)_{L}$ doublet $\left(v_{\tau}, \tau_{L}\right)$, which are composed entirely of unstable particles. Full gauge invariance can be retained if we assume generation-dependent Lorentz-violating parameters $\delta_{e}, \delta_{\mu}$, and $\delta_{\tau}$, for the three $S U(2)_{L}$ doublets, which could be encoded in modified Dirac matrices $\widetilde{\gamma}^{i}=v_{f} \gamma^{i}$ with $f=e, \mu, \tau$ [see Equation (5) of reference [52]]. In the charged-fermion sector, we have nearly no mixing of mass and charge eigenstates. Let us then go into the high-energy regime where, where mass and flavor eigenstates, under the assumptions

$$
\delta_{\mu}, \delta_{\tau}>0, \quad \delta_{\mu} \neq \delta_{\tau}, \quad \delta_{e}=0,
$$

become equal. In this case, at high energy, one would have two neutrino mass eigenstates, which asymptotically approach the muon neutrino and tau neutrino flavor eigenstates at very high energy, decay into electron-positron pairs and (asymptotically) electron neutrinos, via LPCR and NPCR.

Of course, other scenarios and flavor and mass mixing phenomenologies are also possible, as discussed in Sec. IV B of reference [51]. In general, one could interpret the emergence of a specific predominant flavor composition of incoming super-high-energy cosmic neutrinos, consistent with one, and only one, specific mass eigenstate, as a signature of Lorentz violation. This is because a single, defined, oncoming mass eigenstate would be consistent with the two other mass eigenstates being "faster" and thus decaying into the single "slow" eigenstate.

In all discussed scenarios, one might find a conceivable explanation for the apparent cutoff in the cosmic neutrino spectrum at about $2 \mathrm{PeV}$, at the expense of reducing the allowed regime of Lorentz-violating $\delta$ parameters to the range of about $10^{-20}$. In our "attractive scenario," one retains gauge invariance as outlined in Section 4 of reference [52] and still is able to account for a super-high-energy cutoff of the cosmic neutrino spectrum. Experimental confirmation or dismissal of this hypothesis will require better cosmic neutrino statistics at very high energies.

\section{Conclusions}

The existence of the NPCR process (see Figure $1 \mathrm{~b}$ ) reveals a certain dilemma for Lorentz-violating neutrinos (provided the Lorentz violating parameters indicate superluminality). Namely, under the hypothesis of a nonvanishing Lorentz-violating parameter $\delta$, given as in Equation (4), the virtuality

$$
E^{2}-p^{2}=p^{2}\left(v^{2}-1\right) \approx E^{2}\left(v^{2}-1\right)=E^{2} \delta
$$

of a neutrino becomes large for large energy, rendering a number of decay processes kinematically possible. Conversely, based on high-energy astrophysical observations, very strict bounds can be imposed on the Lorentz-violating parameters (see Equations (30)-(32)).

Deep connections exist between Lorentz violation and gauge invariance. In reference [58], it is shown that spontaneous Lorentz violation can lead to an effective low-energy field theory with both Lorentz-breaking and gauge-invariance breaking terms. According to references [58-69], even the photon could potentially be formulated as the Nambu-Goldstone boson linked to spontaneous Lorentz invariance violation. (This ansatz was originally formulated before electroweak unification). 
For a broader view of this point, we refer to Appendix A of reference [52]. If one insists on the persistence of gauge invariance within the electroweak sector, then one has to acknowledge that bounds on Lorentz-violating parameters for charged leptons (e.g., Equation (30)) also apply to the neutrino sector (thus lowering the bound otherwise given in Equation (31) by a factor two, and further restricting the available parameter space for Lorentz-violating parameters in the neutrino sector). Additionally, the assumption that $\delta_{v}=\delta_{e}$ would defeat the purpose of looking at neutrinos for Lorentz violation. If one insists on gauge invariance and still pursues the exploration of Lorentz violation in the neutrino sector, then more sophisticated considerations are required (see Section 4). Namely, one could potentially invoke flavor-dependent differential Lorentz violation across generations (i.e., with different Lorentz-violating parameters for each generation). In this case, flavor and mass eigenstates would become identical in the high-energy limit, and decay and energy loss processes could potentially contribute to an explanation for the apparent cutoff in the cosmic neutrino spectrum in the range of a few PeV (see references [56,57] and the discussion in Section 4).

Funding: This research was funded by National Science Foundation grant number PHY-1710856.

Acknowledgments: The author acknowledges G. Somogyi and I. Nándori.

Conflicts of Interest: The author declares no conflict of interest.

\section{References}

1. Chodos, A.; Hauser, A.I.; Kostelecký, V.A. The Neutrino as a Tachyon. Phys. Lett. B 1985, 150, 431-435. [CrossRef]

2. Jentschura, U.D.; Nándori, I.; Ehrlich, R. Calculation of the Decay Rate of Tachyonic Neutrinos against Charged-Lepton-Pair and Neutrino-Pair Cerenkov Radiation. J. Phys. G 2017, 44, 105201. [CrossRef]

3. Colladay, D.; Kostelecký, V.A. Lorentz-violating extension of the standard model. Phys. Rev. D 1998, 58, 116002. [CrossRef]

4. Kostelecký, V.A. Gravity, Lorentz violation, and the standard model. Phys. Rev. D 2004, 69, 105009. [CrossRef]

5. Kostelecký, V.A.; Mewes, M. Electrodynamics with Lorentz-violating operators of arbitrary dimension. Phys. Rev. D 2009, 80, 015020. [CrossRef]

6. Diaz, J.S.; Kostelecký, V.A.; Mewes, M. Perturbative Lorentz and CPT violation for neutrino and antineutrino oscillations. Phys. Rev. D 2009, 80, 076007. [CrossRef]

7. Kostelecký, V.A.; Mewes, M. Neutrinos with Lorentz-violating operators of arbitrary dimension. Phys. Rev. D 2012, 85, 096005. [CrossRef]

8. Kostelecký, V.A.; Mewes, M. Fermions with Lorentz-violating operators of arbitrary dimension. Phys. Rev. D 2013, 88, 096006. [CrossRef]

9. Diaz, J.S.; Kostelecký, V.A.; Mewes, M. Testing relativity with high-energy astrophysical neutrinos. Phys. Rev. D 2014, 89, 043005. [CrossRef]

10. Diaz, J. Neutrinos as Probes of Lorentz Invariance. Adv. High Energy Phys. 2014, 2014, 962410. [CrossRef]

11. Tasson, J.D. What do we know about Lorentz invariance? Rep. Prog. Phys. 2014, 77, 062901. [CrossRef]

12. Stecker, F.W.; Scully, S.T.; Liberati, S.; Mattingly, D. Searching for traces of Planck-scale physics with high energy neutrinos. Phys. Rev. D 2015, 91, 045009. [CrossRef]

13. Liberati, S. Lorentz symmetry breaking: Phenomenology and constraints. J. Phys. Conf. Ser. 2015, 631, 012011. [CrossRef]

14. Kostelecký, V.A.; Samuel, S. Spontaneous breaking of Lorentz symmetry in string theory. Phys. Rev. D 1989, 39, 683-685. [CrossRef] [PubMed]

15. Kostelecký, V.A.; Potting, R. CPT and Strings. Nucl. Phys. B 1991, 359, 545-570. [CrossRef]

16. Pal, P.B.; Wolfenstein, L. Radiative decays of massive neutrinos. Phys. Rev. D 1982, 25, 766. [CrossRef]

17. Glashow, S.L.; Iliopoulos, J.; Maiani, L. Weak interactions with lepton-hadron symmetry. Phys. Rev. D 1970, 2, 1285. [CrossRef]

18. Amelino-Camelia, G.; Freidel, L.; Kowalski-Glikman, J.; Smolin, L. The principle of relative locality. Phys. Rev. D 2011, 84, 084010. [CrossRef] 
19. Pfeifer, C.; Barcaroli, L.; Brunkhorst, L.; Gubitosi, G.; Loret, N. Hamilton geometry: Phase space geometry from modified dispersion relations. In Proceedings of the 4th Marcel Grossmann Meeting on Recent Developments in Theoretical and Experimental General Relativity, Astrophysics, and Relativistic Field Theories (MG14), Rome, Italy, 12-18 July 2015; pp. 3929-3934.

20. Torri, M.D.C.; Antonelli, V.; Miramonti, L. Homogeneously Modified Special relativity (HMSR). Eur. Phys. J. C 2019, 79, 808. [CrossRef]

21. Amelino-Camelia, G. Relativity: Special treatment (Doubly special relativity). Nature 2002, 418, 34-35. [CrossRef]

22. Magueijo, J.; Smolin, L. Gravity's rainbow. Class. Quantum Grav. 2004, 21, 1725-1736. [CrossRef]

23. Girelli, F.; Liberati, S.; Sindoni, L. Planck-scale modified dispersion relations and Finsler geometry. Phys. Rev. D 2007, 75, 064015. [CrossRef]

24. Magueijo, J.; Smolin, L. Lorentz Invariance with an Invariant Energy Scale. Phys. Rev. Lett. 2002, 88, 190403. [CrossRef] [PubMed]

25. Coleman, S.; Glashow, S.L. High-energy tests of Lorentz invariance. Phys. Rev. D 1999, 59, 116008. [CrossRef]

26. Dadykin, V.L.; Zatsepin, G.T.; Karchagin, V.B.; Korchagin, P.V.; Mal'gin, S.A.; Ryazhskaya, O.G.; Ryasnyi, V.G.; Talochkin, V.P.; Khalchukov, F.F.; Yakushev, V.F.; et al. Detection of a rare event on 23 February 1987 by the neutrino radiation detector under Mont Blanc. JETP Lett. 1987, 45, 593-595.

27. MINOS_Collaboration. Measurement of neutrino velocity with the MINOS detectors and NuMI neutrino beam. Phys. Rev. D 2007, 76, 072005. [CrossRef]

28. Kalbfleisch, G.R.; Baggett, N.; Fowler, E.C.; Alspector, J. Experimental Comparison of Neutrino, Antineutrino, and Muon Velocities. Phys. Rev. Lett. 1979, 43, 1361-1364. [CrossRef]

29. Jentschura, U.D.; Horváth, D.; Nagy, S.; Nándori, I.; Trócsányi, Z.; Ujvári, B. Weighing the Neutrino. Int. J. Mod. Phys. E 2014, 23, 1450004. [CrossRef]

30. Kostelecký, V.A.; Lehnert, R. Stability, causality, and Lorentz and CPT violation. Phys. Rev. D 2001, 63, 065008. [CrossRef]

31. Dubovsky, S.; Nicolis, A.; Trincherini, E.; Villadoro, G. Microcausality in curved space-time. Phys. Rev. D 2008, 77, 084016. [CrossRef]

32. Adam, C.; Klinkhamer, F.R. Causality and CPT violation from an Abelian Chern-Simons like term. Nucl. Phys. B 2001, 607, 247-267. [CrossRef]

33. Reyes, C.M. Causality and stability for Lorentz-CPT violating electrodynamics with dimension-5 operators. Phys. Rev. D 2010, 82, 125036. [CrossRef]

34. Klinkhamer, F.R.; Schreck, M. Consistency of isotropic modified Maxwell theory: Microcausality and unitarity. Nucl. Phys. B 2011, 848, 90-107. [CrossRef]

35. Schreck, M. Analysis of the consistency of parity-odd nonbirefringent modified Maxwell theory. Phys. Rev. D 2012, 86, 06503. [CrossRef]

36. Schreck, M. Quantum field theory based on birefringent modified Maxwell theory. Phys. Rev. D 2014, 89, 085013. [CrossRef]

37. Mankoc Borstnik, N.S. Unification of Spins and Charges. Int. J. Theor. Phys. 2001, 40, 315-337. [CrossRef]

38. Mankoc Borstnik, N.S.; Nielsen, H.B.F. How to generate families of spinors. J. Math. Phys. 2003, 44, 4817-4827. [CrossRef]

39. Mankoc Borstnik, N.S. Can the spin-charge-family theory explain baryon number non conservation? Phys. Rev. D 2015, 91, 065004. [CrossRef]

40. Mankoc Borstnik, N.S.; Nielsen, H.B.F. The spin-charge-family theory offers understanding of the triangle anomalies cancellation in the standard model. Prog. Phys. 2016, 65, 1700046.

41. Mankoc Borstnik, N. The Spin-Charge-Family theory offers the explanation for all the assumptions of the Standard model, for the Dark matter, for the Matter-antimatter asymmetry, making several predictions. In Proceedings of the Conference on New Physics at the Large Hadron Collider, Singapore, 29 February-4 March 2016; Fritzsch, H., Ed.; World Scientific: Singapore, 2017; pp. 161-194.

42. Bojowald, M. Canonical Gravity and Applications; Cambridge University Press: Cambridge, UK, 2011.

43. Jentschura, U.D.; Noble, J.H. Nonrelativistic Limit of the Dirac-Schwarzschild Hamiltonian: Gravitational Zitterbewegung and Gravitational Spin-Orbit Coupling. Phys. Rev. A 2013, 88, 022121. [CrossRef]

44. Jentschura, U.D. Gravitational Effects in $g$ Factor Measurements and High-Precision Spectroscopy: Limits of Einstein's Equivalence Principle. Phys. Rev. A 2018, 98, 032508. [CrossRef] 
45. Arias, P.; Gamboa, J.; Lopez-Sarrion, J.; Mendez, F.; Das, A. CPT/Lorentz invariance violation and neutrino oscillation. Phys. Lett. B 2007, 650, 401-406. [CrossRef]

46. Liberati, S. Tests of Lorentz invariance: A 2013 update. Class. Quantum Grav. 2013, 30, 133001. [CrossRef]

47. Antonelli, V.; Miramonti, L.; Torri, M.D.C. Neutrino oscillations and Lorentz invariance violation in a Finslerian geometrical model. Eur. Phys. J. C 2018, 78, 667. [CrossRef]

48. Torri, M.D.C. Neutrino Oscillations and Lorentz Invariance Violation. Universe 2020, 6, 37. [CrossRef]

49. Cohen, A.G.; Glashow, S.L. Pair Creation Constrains Superluminal Neutrino Propagation. Phys. Rev. Lett. 2011, 107, 181803. [CrossRef] [PubMed]

50. Bezrukov, F.; Lee, H.M. Model dependence of the bremsstrahlung effects from the superluminal neutrino at OPERA. Phys. Rev. D 2012, 85, 031901(R). [CrossRef]

51. Somogyi, G.; Nándori, I.; Jentschura, U.D. Neutrino Splitting for Lorentz-Violating Neutrinos: Detailed Analysis. Phys. Rev. D 2019, 100, 035036. [CrossRef]

52. Jentschura, U.D.; Nándori, I.; Somogyi, G. Lorentz Breaking and $S U(2)_{L} \times U(1)_{Y}$ Gauge Invariance for Neutrino Decays. Int. J. Mod. Phys. E 2019, 28, 1950072. [CrossRef]

53. Byckling, E.; Kajantie, K. Particle Kinematics; John Wiley \& Sons: New York, NY, USA, 1973.

54. Stecker, F.W. Limiting superluminal electron and neutrino velocities using the 2010 Crab Nebula flare and the IceCube PeV neutrino events. Astropart. Phys. 2014, 56, 16-18. [CrossRef]

55. Stecker, F.W.; Scully, S.T. Propagation of superluminal PeV IceCube neutrinos: A high energy spectral cutoff of new constraints on Lorentz invariance violation. Phys. Rev. D 2014, 90, 043012. [CrossRef]

56. IceCube_Collaboration. First Observation of PeV-Energy Neutrinos with IceCube. Phys. Rev. Lett. 2013, 111, 021103. [CrossRef] [PubMed]

57. IceCube_Collaboration. Observation of High-Energy Astrophysical Neutrinos in Three Years of IceCube Data. Phys. Rev. Lett. 2014, 113, 101101. [CrossRef] [PubMed]

58. Chkareuli, J.L.; Jejeleva, J.G. Spontaneous Lorentz violation: Non-Abelian gauge fields as pseudo-Goldstone vector bosons. Phys. Lett. B 2008, 659, 754-760. [CrossRef]

59. Heisenberg, W. Quantum Theory of Fields and Elementary Particles. Rev. Mod. Phys. 1957, 29, $269-278$. [CrossRef]

60. Bjorken, J.D. A Dynamical Origin for the Electromagnetic Field. Ann. Phys. 1963, 24, 174-187. [CrossRef]

61. Bialynicki-Birula, I. Quantum Electrodyanmics without Electromagnetic Field. Phys. Rev. 1963, 130, 465-468. [CrossRef]

62. Eguchi, T. New approach to collective phenomena in superconductivity models. Phys. Rev. D 1976, 14, 2755-2763. [CrossRef]

63. Chkareuli, J.L.; Froggatt, C.D.; Nielsen, H.B. Lorentz Invariance and Origin of Symmetries. Phys. Rev. Lett. 2001, 87, 091601. [CrossRef]

64. Chkareuli, J.L.; Froggatt, C.D.; Nielsen, H.B. Spontaneously generated gauge invariance. Nucl. Phys. B 2001, 609, 46-60. [CrossRef]

65. Bjorken, J.D. Emergent Gauge Bosons. arXiv 2001, arXiv:hep-th/0111196.

66. Azatov, A.T.; Chkareuli, J.L. Nonlinear QED and physical Lorentz invariance. Phys. Rev. D 2006, 73, 065026. [CrossRef]

67. Chkareuli, J.L.; Kepuladze, Z.R. Nonlinear massive QED and physical Lorentz invariance. Phys. Lett. B 2007, 644, 212-217. [CrossRef]

68. Chkareuli, J.L.; Froggatt, C.D.; Jejeleva, J.G.; Nielsen, H.B. Constrained gauge fields from spontaneous Lorentz violation. Nucl. Phys. B 2008, 796, 211-223. [CrossRef]

69. Chkareuli, J.L.; Froggatt, C.D.; Nielsen, H.B. Deriving Gauge Symmetry and Spontaneous Lorentz Violation. Nucl. Phys. B 2009, 821, 65-73. [CrossRef]

(c) 2020 by the author. Licensee MDPI, Basel, Switzerland. This article is an open access article distributed under the terms and conditions of the Creative Commons Attribution (CC BY) license (http:/ / creativecommons.org/licenses/by/4.0/). 